\title{
Serine/Threonine-Protein Phosphatase 2A Regulatory Subunit B" Subunit Beta
}

National Cancer Institute

\section{Source}

National Cancer Institute. Serine/Threonine-Protein Phosphatase 2A Regulatory Subunit

B" Subunit Beta. NCl Thesaurus. Code C37317.

Serine/threonine-protein phosphatase 2A regulatory subunit B" subunit beta (575 aa,

$\sim 65 \mathrm{kDa}$ ) is encoded by the human PPP2R3B gene. This protein may be involved in the localization, substrate specificity and the modulation of activity for protein phosphatase $2 \mathrm{~A}$. 